\title{
Wireless Monitoring in Intensive Care Units by a 3D-Printed System with Embedded Electronic
}

\author{
Flavia Basilotta ${ }^{\mathrm{a}, \mathrm{b}}$, Stefano Riario ${ }^{\mathrm{a}}$, Francesca Stradolini ${ }^{\mathrm{a}}$, Irene Taurino ${ }^{\mathrm{a}}$, \\ Danilo Demarchi $^{\mathrm{b}}$, Giovanni De Micheli ${ }^{\mathrm{a}}$, Sandro Carrara ${ }^{\mathrm{a}}$ \\ ${ }^{a}$ Laboratory of Integrated Systems, Swiss Federal Institute of Technology (EPFL), Lausanne, Switzerland \\ ${ }^{\mathrm{b}}$ Department of Electronics and Telecommunications, Politecnico di Torino, Torino, Italy
}

\begin{abstract}
In this paper, the design, realization, and preliminary testing of a portable wireless system for measuring key metabolites (e.g., glucose, lactate, calcium, potassium) in intensive care monitoring is presented. The system consists of a 3D-Printed case, which includes the fluidic system that drives the monitored human fluids on top of the sensing devices. The case fully integrates a hardware platform on PCB (Printed Circuit Board) that connects the biosensors to the read-out font-end and to a Bluetooth $^{\circledR}$ module for the data transmission to a mobile Android interface. Two metabolites, glucose and lactate, that are important to monitor in critical patients, were measured in the range of clinical interest.
\end{abstract}

Keywords-3D-printing; Biosensors; microfluidic chamber; Android; Smart device.

\section{INTRODUCTION}

Continuous health monitoring for patients in Intensive Care Unit (ICU) is a key factor for an appropriate and an immediate emergency intervention when necessary [1]. The level in the patient's fluids (e.g., the blood) of several metabolites, like glucose and lactate [2], and ions, like sodium and potassium [3], are key parameters to be continuously monitored in order to react promptly in case of sudden degeneration toward a multiple organ failure, which might be fatal for the patient. Electrochemical sensors can be exploited in order to measure those parameters for ICU diseases. In fact, they have been already largely developed and successfully used for measurement of both endogenous (glucose [4], lactate [5], cholesterol [6]) and exogenous (anticancer [7] or antiinflammatory [8] drugs) metabolites as well as for ions detection (calcium [9], and potassium [10]). Moreover, they are definitely suitable for highly miniaturized integration (in implantable devices [11]).

In this paper, we present the first prototype of a definitely novel smart portable device that can be used as a fully noninvasive fluidic feedback control unit for continuous monitoring of critical patients. The full device is constituted by a very small box (few $\mathrm{cm}^{3}$ ), which contains components all required for the monitoring: a fluidic chamber to flow the patient's fluid on the sensors; a passive chip with a series of electrochemical sensors for endogenous metabolites and ions; a system on board for reading the sensors, a Bluetooth ${ }^{\circledR}$ module for data transmission; a lithium rechargeable battery that provides power to the whole system. The system is then connected, via Bluetooth ${ }^{\circledR}$, to a tablet (or a smartphone) and visualizes and processes the received data thanks to a novel developed user-friendly Android interface. This paper presents the preliminary test done on the monitoring of two endogenous metabolites (glucose and lactate) with the first prototype of the integrated system.

\section{The Fluidic DeVICE}

\section{Design a fabrication of micro-fluidic chamber}

The microfluidic system was designed to minimize the crosstalking phenomenon between WE1, WE2 and WE3, where lactate and glucose detection takes place. In amperometric biosensors chemical cross talk exists due to the diffusion of electro-active substances generated by enzymatic reactions at one electrode to the adjacent ones. This phenomenon deteriorates the specificity and alters the monitoring values.

Before the fabrication of the microfluidic chamber, Fluid dynamic and Diffusion of chemical species simulations were run by Comsol Multiphisics ${ }^{\circledR}$ software; in this step the geometry and the dimension of the chamber, the inlet flow rate $(13 \mu \mathrm{l} / \mathrm{min})$, the position and the dimension of inlet and outlet were chosen to provide a laminar flow regime, preventing the cross-talk phenomenon.

Fig. 1 shows a Comsol simulation, where the streamlines mimic the laminar fluid and the color mimics the chemical reaction and the $\mathrm{H}_{2} \mathrm{O}_{2}$ produced following the enzymatic reaction. The laminar regime inside the chamber was confirmed in laboratory using a 3D-printed prototype only of the chamber, which returned the same result, as shown in Fig. 2. The black streamlines pass through the three small electrodes, WE1, WE2 and WE3, without any turbulent behavior. It was verified, computationally and experimentally, that the decrease of one dimension $\left(10^{-1} \mathrm{~m} / \mathrm{s}\right)$ of the inlet flow rate and the increase of just $1 \mathrm{~m}^{2} / \mathrm{s}$ of $\mathrm{H}_{2} \mathrm{O}_{2}$ coefficient diffusion produce a high cross-talking $\left(\mathrm{H}_{2} \mathrm{O}_{2}\right.$ spreads onto the whole platform, not just near the WEs). Afterwards, a virtual design of the fluidic system was implemented in the sldprt format by Solidworks ${ }^{\circledR}$ software (SW). Fig. 3a shows the SW final fluidic system design, while Fig. 4 was the implementation of the entire device case embedding the fluidic system too. 


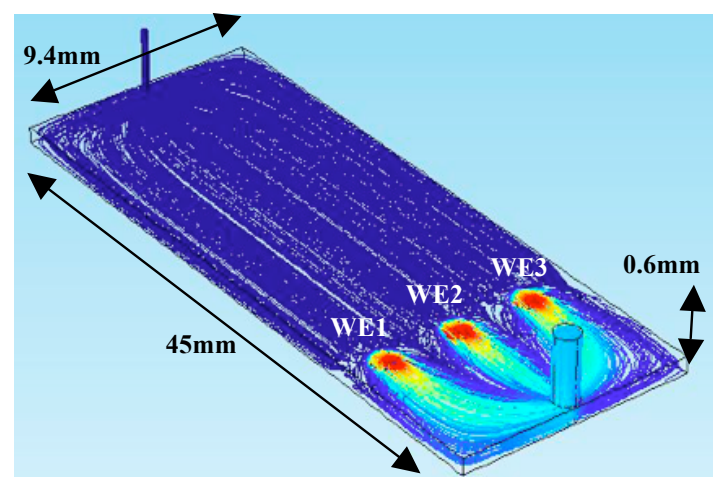

Fig. 1: Laminar flow and diffusion simulation. The hydrogen peroxide generated onto WEs (in red) is spread toward the output following the flow laminar regime.

The CAD model was then printed using the Multijet Modelling (MM) method at Atelier de Fabrication Additive (AFA) of École Polytechnique Fédérale de Lausanne (EPFL) while several models of the fluidic chambers were realized by using Stereolithography method at Proform AG enterprise in Fribourg due to the different materials considered for the different prototypes. In fact, the fluidic system model was realized in two different materials depending on the printing technique: MM or Stereolitography. The performances of the realized fluid chambers were investigated at first under flow condition and then in multidetection analysis. The thermoplastic polymer VeroWhite was used as material when MM printing technique was employed. Unfortunately, the developed model surface was too rough to guarantee a laminar flow regime inside the chamber (the streamlines were perturbed between the fluid layers) due to printer resolution ( $0.3 \mathrm{~mm})$. By Stereolitography method, the liquid photopolymer, Expoxy translucent, was used as material. Printing resolution was finer $(\sim 0.1 \mathrm{~mm})$ than $\mathrm{MM}$ one so less layer perturbation occurred, thus better detection was achieved. However, in both cases, it was not possible to achieve a straight laminar flow regime due to materials corrugation in the outlet region. To cope with this problem, two printed walls were introduced in order to mechanically separate WE1, WE2, and WE3. The final case as obtained by the MM printing technique is shown in Fig. 4.

\section{The SENSING Platform}

\section{A. Platform Microfabrication}

The micro-fabrication of the passive chip was realized with a two-mask process flow. The platform measures $9.3 \times 35 \mathrm{~mm}$ and it hosts an array of three working electrodes (WEs), a counter electrode (CE) and a reference electrode (RE), all made in Pt. The radius of three working electrodes (WE1, WE2, WE3 on the right in Fig.1) used for the measurements of the present work, is $282 \mu \mathrm{m}$. Fig. 4 also shows the location of such passive platform once integrated in the whole device. The fluidic chamber (shown on the top-right of Fig. 4) is then gently inserted in order to hosts the passive chip and to assure the fit with the electrochemical sensors.

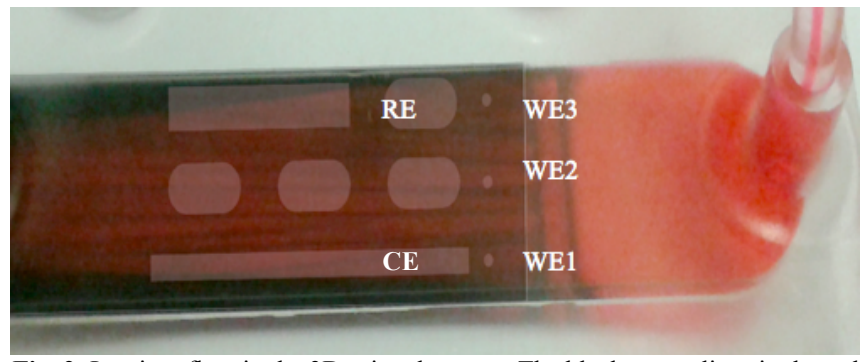

Fig. 2: Laminar flow in the 3D printed system. The black streamlines in the red solution perform the blue streamlines computationally simulated in Fig.1.

\section{B. Sensors Nanostructuration}

Electrochemical behavior of the three-cell sensor was improved by the electrodeposition of $\mathrm{Ag}$ on the RE. Pt nanospheres were electrodeposited on WE1, WE2 and WE3 to increase the surface area of the working electrodes, to improve the catalytic performance and for a longer restrain of the threelayers of enzyme depositions. More details on the nanostructuration can be found in [12].

\section{Sensors bio-functionalization}

Glucose oxidase (GOx) from Aspergillus Niger and lactate oxidase (LOx) from Pediococcus species were purchased from Roche in lyophilizate powder and dissolved in a $10 \mathrm{mM}$ Phosphate Buffered Saline solution (PBS, pH 7.4), with the addition of glutaraldehyde $0.25 \%$. Glucose and lithium Llactate were purchased from Sigma-Aldrich (Switzerland) in powder and dissolved in PBS, $\mathrm{pH}$ 7.4. For the glucose and lactate detection, $1 \mu \mathrm{l}$ of a solution $(15 \mathrm{mg} / \mathrm{ml}$ of GOx and 100 $\mathrm{mg} / \mathrm{ml}$ of LOx) were drop cast three times on two different nanostructured WEs. Electrodes were stored overnight at $4^{\circ} \mathrm{C}$. All samples were freshly prepared and used the same day.

\section{THE ELECTRONICS}

\section{A. PCB System}

The PCB contains a mixed signal integrated circuit (LMP91000) provided by Texas Instruments, which main blocks are:

1. The potentiostat: in its classic configuration with an operational amplifier which is able to keep the voltage drop between the reference and the working electrode $\left(\mathrm{V}_{\text {bias }}\right)$ constant by automatically changing the voltage on the CE.

2. The transimpedance amplifier: able to convert the value of the current flowing inside the electrochemical cell collected by the working electrode (WE) into a voltage by the relation

$$
V_{\text {out }}=I_{\text {cell }}-R_{\text {TIA }}+V_{\text {zero }} \text {. }
$$

3. The digital $\mathrm{I}^{2} \mathrm{C}$ (Inter-Integrated Circuit) interface: with the $\mathrm{I}^{2} \mathrm{C}$ digital transmission protocol, is able to receive commands from outside in order to read or write inside the 8 bits registers. By changing the values of those registers, it is possible to modify the value of $\mathrm{V}_{\text {bias }}$ from $0 \%$ to $14 \%$ of the voltage reference, which can be the voltage supply or external, the $\mathrm{V}_{\text {zero }}$ as the $20 \%, 50 \%$ or $67 \%$ of the voltage reference, and to choose over seven different values of the RTIA. 
a)
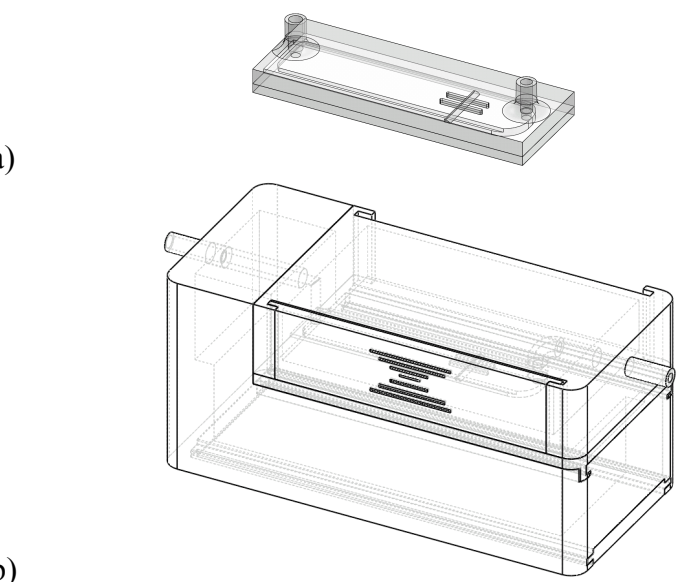

Fig 3: a) Microfluidic chamber only; b) The designed full case that includes the fluidic systems

For more features see the LMP91000 datasheet [13]. All the commands are provided by the Atmel microcontroller ATTiny 1634 using the embedded and very versatile Universal Serial Interface (USI) able to provide the $\mathrm{I}^{2} \mathrm{C}$ protocol. The internal 10 bit $\mathrm{ADC}$ is used to convert the analog signal $\mathrm{V}_{\text {out }}$ that contains the information of the current flowing into the electrochemical cell in digital words that are packed in two bytes and transmitted by the UART interface to the Roving's Bluetooth ${ }^{\circledR}$ module RN-42. The microcontroller is also driving the analog multiplexer MAX4734 in order to select the desired working electrode. A schematic of the hardware is depicted in Fig. 5. In average, the whole system consumes $30 \mathrm{~mA}$ at $3.7 \mathrm{~V}$, while the Bluetooth module requires almost the entire power demand during data transmission. Power is supplied by a lithium rechargeable battery (model EFLB5001S25, with capacity of $500 \mathrm{~mA} \mathrm{~h}$ ).

\section{A. Wireless interface}

The main aim of the Android interface is the immediate display of the measured values on the monitor of tablets or smartphones without waiting for the time-consuming $a$ posteriori analysis. Briefly, the wireless communication between hardware platform and mobile interface is enabled using the Bluetooth ${ }^{\circledR}$ technology which is standard, economic and secure.

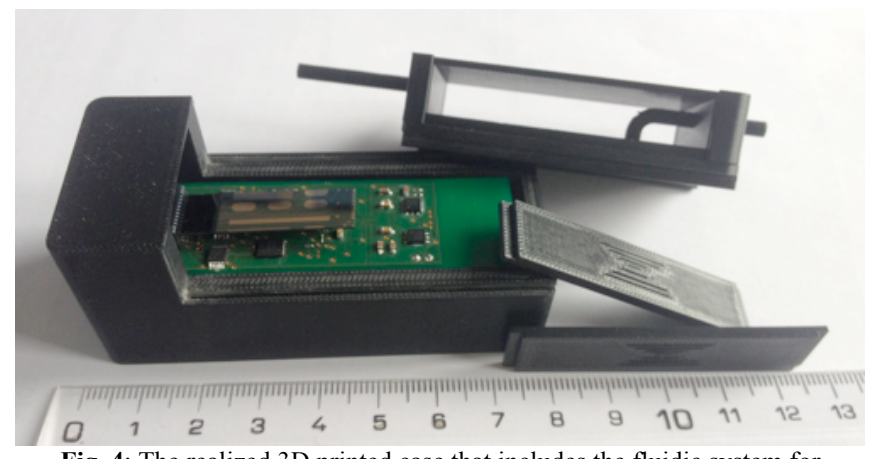

Fig. 4: The realized 3D printed case that includes the fluidic system for continuous monitoring in ICUs

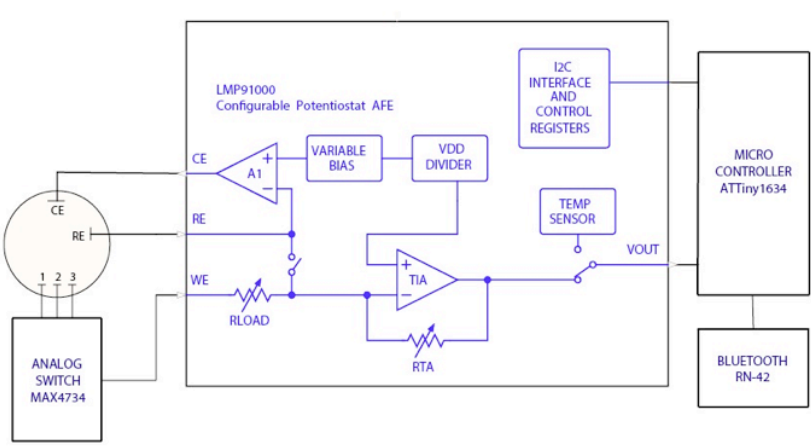

Fig 5: Hardware Schematic

A Service keeps active the exchange of data between these two devices even if the application is running in background. The interface consists of four principal Activities:

(1) Welcome, (2) Settings, (3) Calibration, (4) Monitoring.

The Welcome Activity is shown as soon as the interface is opened. It enables the user to choose up to seven electrodes to monitor The Settings Activity offers the possibility to modify some parameters of the potentiostat: transimpedance gain $\left(\mathrm{R}_{\mathrm{TIA}}\right)$, load resistance $\left(\mathrm{R}_{\mathrm{LOAD}}\right)$, internal zero current, bias and bias sign. Moreover it is also possible to verify in continuous monitoring mode if the chosen parameters are suitable for the hardware. The Calibration Activity guides the user in the calibration process of all sensors of the platform. The user has just to choose the electrode to be calibrated and to follow the guide. Both Calibration and Settings Activities are accessible only by specialist user through a login. Finally, in the Monitoring Activity the main screen shows as many charts as the monitored electrodes. Every time a new data is received from the sensor, plots are automatically updated. All the received data are stored in files in the memory of the mobile device so that the user can consult them when necessary. Moreover, a moving average filter with a programmable action window and a median filter are available for filtering the received data. A deeper and more detailed description of the Android interface can be found in [14].

\section{SYSTEM TESTS}

\section{A. Electrochemical tests}

We performed electrochemical measurements of glucose and lactate. The testing set-up consisted of an enzymefunctionalized platform well sealed into the printed microfluidic chamber to prevent leakage risks. The inlet and outlet of the chamber were connected to PVC pipes of $0.25 \mathrm{~mm}$ ID; the inlet pipe was bond to a peristaltic pump, working at 3 revolutions per minute (RPM) so that the sample solution circulated with a velocity of around $13 \mu \mathrm{l} / \mathrm{min}$. Then the platform was connected to an Autolab electrochemical workstation (Metrohm Switzerland) by a multiplexer. 


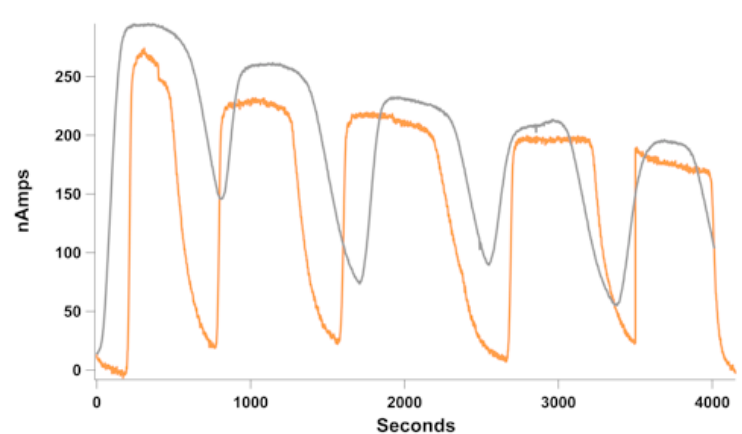

Fig. 6: Chronoamperometry for glucose (grey) and lactate (orange)

Chronoamperometry measures were carried out by keeping a constant potential of $+0.7 \mathrm{~V}$ between the $\mathrm{RE}$ and the chosen WE. Due to the applied potential, $\mathrm{H}_{2} \mathrm{O}_{2}$ is electro-oxidized and an increase of the current is recorded and is proportionally related to the analyte concentration in the probing solution. Glucose and lactate calibrations were performed, changing the inlet solutions from $1 \mathrm{mM}$ to $9 \mathrm{mM}$, with $1 \mathrm{mM}$ step, starting from a PBS baseline. Once measurement started, we changed the solution at the inlet of the fluidic circuit with an interval time of $500 \mathrm{~s}$.. For glucose the sensitivity results in a value of $105.16 \mathrm{nA} / \mathrm{mM} \mathrm{mm}^{-2}$ (standard error $=29.9, \mathrm{R}^{2}=0.95$ ). While for lactate the obtained sensitivity is $51.43 \mathrm{nA} / \mathrm{mM} \mathrm{mm}^{-2}$ ( standard error $=24.7, \mathrm{R}^{2}=0.89$ ). Six glucose calibrations and four lactate calibrations were performed in order to assure statistics in sensor characterization. Two examples of time trends acquired by using the full device are shown in Fig. 6. All these experiments were carried out in a $10 \mathrm{mM}$ PBS (pH 7.4) as supporting electrolyte.

\section{CONCLUSION}

We designed, built, and tested a system based on a 3D-printed case that includes a microfluidic chamber, a passive chip hosting several electrochemical sensors, an electronic system on board that assures sensors reading as well as data communication. The microfluidic chamber was designed in order to minimize the cross talking among three electrodes. To further reduce the cross talking among different sensors, microwalls have been also added in the region of the inlet. The system was also connected to an android interface (typically a tablet) for immediate visualization of the acquired time trend on the monitored parameter. To test the whole system, the sensing of glucose and lactate was performed both to calibrate the sensors (to characterize their sensitivity) as well as to demonstrate the system capability for continuous monitoring. The Android interface, running on a tablet, shown instantaneously the acquired value for glucose and lactate meanwhile a liquid current was flowing in the fluidic chamber hosting the electrochemical sensors. The obtained results were comparable with similar ones acquired by using a commercially available lab potentiostat. Therefore, we demonstrated that the realization of extremely small and portable devices with wireless communication for monitoring the concentration of endogenous metabolites, which are also critical parameters for patients in intensive care units, is actually feasible. To this end, we used very cheap off-the-shelf standard electronic components and a 3D-printed packaging to obtain the case as well as the required fluidics.

\section{ACKNOWLEDGEMENTS}

The research received funding by the IronIC++ project (Swiss Nano-Tera.ch initiative granted by the Swiss National Science Foundation).

\section{REFERENCES}

[1] Philip A. Goldberg, Mark D. Siegel, Raymond R. Russell, Robert S. Sherwin,Joshua I. Halickman, Dawn A. Cooper, James D. Dziura, and Silvio E. Inzucch., Experience with the Continuous Glucose Monitoring System in a Medical Intensive Care Unit.,Diabetes Technology andTherapeutics, Vol.6, July 2004, Pages. 339 - 347.

[2] D.A. Jones, M.C. Parkin, H. Langemann, H. Landolt, S.E. Hopwood, A.J. Strong and M.G. Boutelle. On-line monitoring in neurointensive care : Enzyme-based electrochemical assay for simultaneous, continuous monitoring of glucose and lactate from critical care patients., Journal of Electroanalytical Chemistry, 538(2002) 243-252.

[3] Johnston, Andrew J., and Arun K. Gupta. Advanced monitoring in the neurology intensive care unit: microdialysis, Current opinion in critical care 8(2002) 121-127.

[4] Wang, J, Electrochemical glucose biosensors, Chemical reviews 10 (2008) 814-825

[5] Poscia, A., Messeri, D., Moscone, D., Ricci, F., \& Valgimigli, F., A novel continuous subcutaneous lactate monitoring system, Biosensors and bioelectronics, 20(2005) 2244-2250

[6] Carrara S., Shumyantseva V.V., Archakov A.I., Samorì, B. ScreenPrinted Electrodes based on Carbon Nanotubes and Cytochrome P450scc for Highly-Sensitive Cholesterol Biosensors, Biosensors and Bioelectronics, 24(2008) 148-150

[7] Carrara A., Cavallini A., Erokhin V., De Micheli G., Multi-panel drugs detection in human serum for personalized therapy, Biosensors and Bioelectronics, 26 (2011) 3914-3919

[8] Baj-Rossi C., De Micheli G., Carrara S., Continuous monitoring of Naproxen by a cytochrome P450-based electrochemical sensor, Biosensors and Bioelectronics, 53(2014) 283-287

[9] Zhang, X., Wang, S., Jia, L., Xu, Z., \& Zeng, Y., An electrochemical sensor for determination of calcium dobesilate based on PoPD/MWNTs composite film modified glassy carbon electrode, Journal of biochemical and biophysical methods, 70(2008) 1203-1209.

[10] Růžička, J., E. H. Hansen, \& E. A. Zagatto. Flow injection analysis: Part VII. Use of ion-selective electrodes for rapid analysis of soil extracts and blood serum. Determination of potassium, sodium and nitrate. Analytica Chimica Acta 88.1 (1977): 1-16.

[11] Baj-Rossi C., Kilinc E.G., Ghoreishizadeh S.S., Casarino D., Rezzonico Jost T., Dehollain C., Grassi F., Pastorino L., De Micheli G. , Carrara S., Full Fabrication and Packaging of an Implantable Multi-panel Device for Monitoring of Metabolites in Small Animals, IEEE Transaction on Biomedical Circuit and Systems, TBCAS 8(2014) 636-647

[12] Taurino I. and Sanzo G., Mazzei F., Favero G., De Micheli G., Carrara S., Fast synthesis of platinum nanopetals and nanospheres for highlysensitive non-enzymatic detection of glucose and selective sensing of ions, Submitted to Scientific Report

[13] Texas Intruments, "LMP91000 Sensor AFE System: Configurable AFE Potentiostat for Low-Power Chemical Sensing Applications", (http://www.ti.com/lit/ds/symlink/lmp91000.pdf), March 2013.

[14] Stradolini F., Riario S., Boero C., Baj-Rossi C., Taurino I., Surrel G., De Micheli G., Carrara S., Wireless Monitoring of Endogenous and Exogenous Biomolecules on an AndroidTM Interface, Submitted to IEEE Sensors 